\title{
A unified approach of catastrophic events
}

\author{
S. Nikolopoulos ${ }^{1}$, P. Kapiris ${ }^{2}$, K. Karamanos ${ }^{3}$, and K. Eftaxias ${ }^{2}$ \\ ${ }^{1}$ National Technical Univ. Athens, Dept. of Electrical \& Computer Engineering Zografou Campus, Zografou 15773, Greece \\ ${ }^{2}$ Dept. of Solid State Section, Faculty of Physics, Univ. of Athens Panepistimioupolis Zografou, 15784 Athens, Greece \\ ${ }^{3}$ Centre for Nonlinear Phenomena and Complex Systems, Univ. Libre de Bruxelles, CP 231, Campus Plaine, B-1050 \\ Brussels, Belgium
}

Received: 20 July 2004 - Revised: 8 October 2004 - Accepted: 11 October 2004 - Published: 14 October 2004

Part of Special Issue "Precursory phenomena, seismic hazard evaluation and seismo-tectonic electromagnetic effects"

\begin{abstract}
Although there is an accumulated charge of theoretical, computational, and numerical work, like catastrophe theory, bifurcation theory, stochastic and deterministic chaos theory, there is an important feeling that these matters do not completely cover the physics of real catastrophic events. Recent studies have suggested that a large variety of complex processes, including earthquakes, heartbeats, and neuronal dynamics, exhibits statistical similarities. Here we are studying in terms of complexity and non linear techniques whether isomorphic signatures emerged indicating the transition from the normal state to the both geological and biological shocks. In the last 15 years, the study of Complex Systems has emerged as a recognized field in its own right, although a good definition of what a complex system is, actually is eluded. A basic reason for our interest in complexity is the striking similarity in behaviour close to irreversible phase transitions among systems that are otherwise quite different in nature. It is by now recognized that the pre-seismic electromagnetic time-series contain valuable information about the earthquake preparation process, which cannot be extracted without the use of important computational power, probably in connection with computer Algebra techniques. This paper presents an analysis, the aim of which is to indicate the approach of the global instability in the prefocal area. Non-linear characteristics are studied by applying two techniques, namely the Correlation Dimension Estimation and the Approximate Entropy. These two non-linear techniques present coherent conclusions, and could cooperate with an independent fractal spectral analysis to provide a detection concerning the emergence of the nucleation phase of the impending catastrophic event. In the context of similar mathematical background, it would be interesting to augment this description of pre-seismic electromagnetic anomalies in order to cover biological crises, namely, epileptic seizure and heart failure.
\end{abstract}

Correspondence to: K. A. Eftaxias

(ceftax@phys.uoa.gr)

\section{Introduction}

Prediction of natural phenomena has always been a wellpondered problem. In physics, the predictability degree of a phenomenon is often measured by how well we understand it. Despite the large amount of experimental data and the considerable effort that has been undertaken by the material scientists, many questions about the fracture remain standing.

When a heterogeneous material is strained, its evolution toward breaking is characterized by the nucleation and coalescence of micro-cracks before the final break-up. Both acoustic as well as electromagnetic (EM) emission in a wide frequency spectrum ranging from very low frequencies (VLF) to very high frequencies (VHF), is produced by micro-cracks, which can be considered as the so-called precursors of general fracture. These precursors are detectable both at a laboratory and a geological scale. Several experimental results, which illustrate the connection between anomalous VLF-VHF electromagnetic phenomena and acoustic phenomena with earthquake preparation, were presented in a rather comprehensive collection of papers edited by (Hayakawa and Fujinawa, 1994; Hayakawa, 1999; Hayakawa and Molchanov, 2002).

Aiming at recording VLF-VHF electromagnetic precursors, since 1994 a station was installed at a mountainous site of Zante island $\left(37.76^{\circ} \mathrm{N}-20.76^{\circ} \mathrm{E}\right)$ in western Greece (Fig. 1).

An important earthquake (Ms=5.9) occurred on 7 September 1999 at 11:56 GMT at a distance of about $20 \mathrm{~km}$ from the center of the city of Athens, the capital of Greece. Very clear electromagnetic anomalies have been detected in the VLF band (Fig. 2), i.e. at $3 \mathrm{kHz}$ and $10 \mathrm{kHz}$, before the Athens EQ (Eftaxias et al., 2000, 2001). The whole EM precursors were emerged from 31 August to 7 September 1999 (Fig. 2). It is characterized by an accelerating emission rate (Fig. 2), while, this radiation is embedded in a long duration quiescence period concerning the detection of EM disturbances at the VLF frequency band. These emissions have a rather 


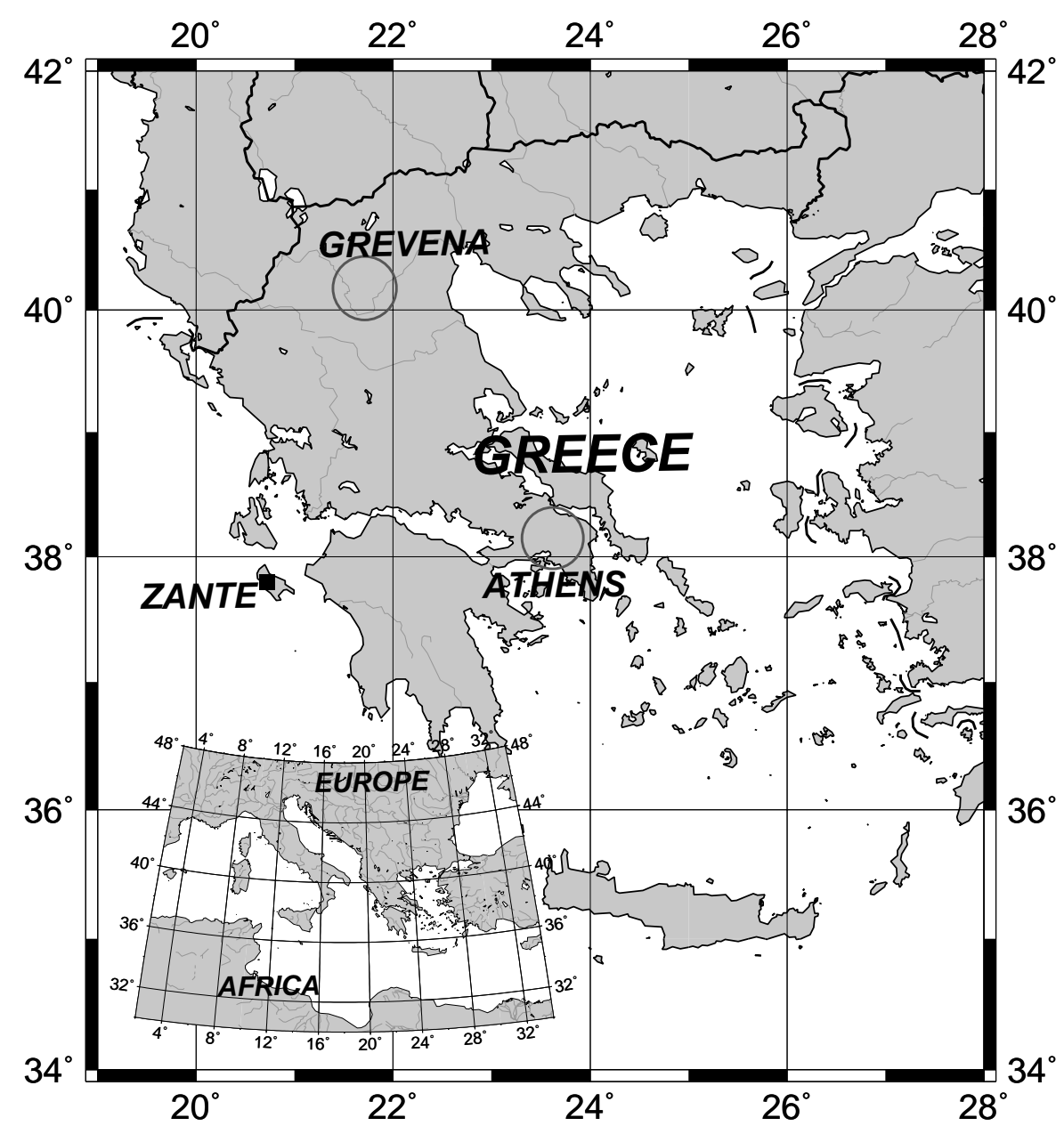

Fig. 1. The map demonstrates the location of the Zante RF station $(\bigcirc)$.

long duration, (the data were sampled at $1 \mathrm{~Hz}$ ), and thus it provides sufficient data for statistical analysis.

Recently, in a series of papers (Eftaxias et al., 2001, 2002; Kapiris et al., 2003, 2004b,a; Eftaxias et al., 2004), we attempt to establish the hypothesis that the pre-seismic electromagnetic emissions offer a potential window for a step by step monitoring of the last stages of earthquake preparation processes. However, it is difficult to prove association between any two events (possible precursor and earthquake) separated in times. As a major result, the present study indicates that it seems useful to combine various computational methods to enhance the association of the pre-seismic EM phenomena with micro-fracturing in the pre-focal area. The achievement of converging estimations would definitely improve the chances for an understanding of the physics behind the generation of earthquakes.

\section{Background information}

In this section, we briefly describe the algorithms that were used and compared in this study. Their main characteristics as well as the reasons they were chosen are discussed.
) and the epicentres of the Athens and Kozani-Grevena earthquakes

\subsection{The Delay Times Method}

The Delay Times method is an important tool in non-linear analysis and gives both a qualitative and quantitative measure of the complexity of the time-series under examination. It was first established by (Grassberger and Procaccia, 1983) and is based on the Takens Theorem (Takens, 1981). A timeseries is constructed from a set of successive and experimentally derived values. From the original time-series we then construct a new series, which in this case is composed of vectors. For the construction of each of the vectors the estimation of two parameters, the embedding dimension, $m$, and the time lag, $\tau$, is required. The time lag represents the window that is used for the computation of the coordinates of these vectors. It is estimated from the decorrelation time, which is the window beyond which the signal ceases to present periodicities. The decorrelation time is calculated either from the first zero-value of the autocorrelation function, or from the first value of the mutual information function (Farmer and Swinney, 1986) that is close to zero. The mutual information function is a widely accepted method that computes non-linear and linear correlation of a signal. The 


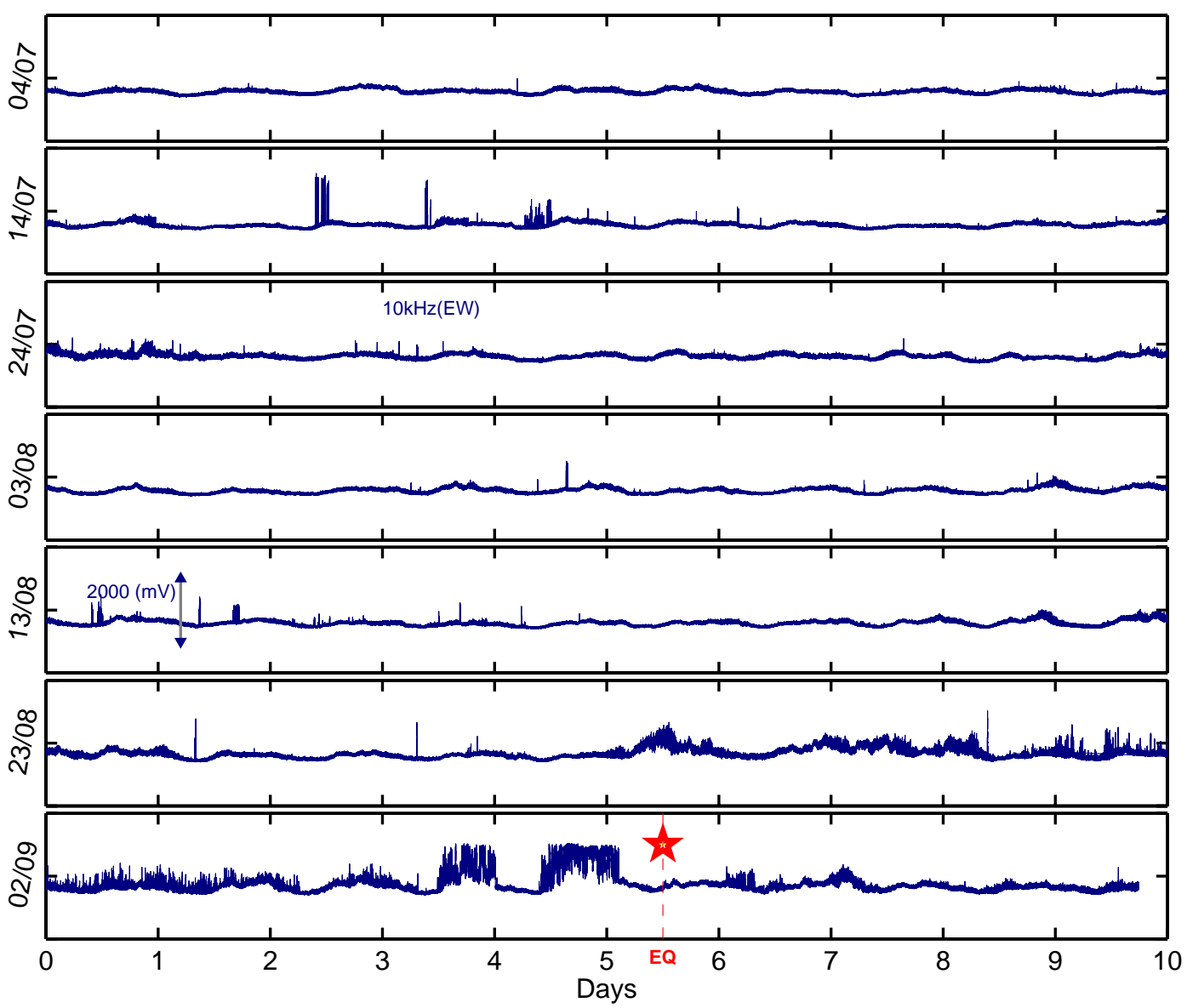

Fig. 2. Time-series of the $10 \mathrm{kHz}(\mathrm{E}-\mathrm{W})$ magnetic field strength between 4 July 1999 and 11 September 1999 in arbitrary units. The precursory accelerating emission is embedded in a long duration of quiescence period. The star indicate the time of the Athens earthquake occurrence.

parameter $m$ is assigned increasing integer values, in a range that satisfies both the Takens criterion and the maximum admitted window length, according to basic non-linear dynamics theory. Appendix A includes analytical information on these parameters, as well as a detailed description of the entire Delay Times method.

Once the above is completed, the correlation integral, $C(r)$, is computed for increasing values of $r$. This integral basically computes how many of the above vectors have a distance between them less than $r$, where $r$ is a ray in the vector space. We are then able to plot $\ln (C)$ vs. $\ln (r)$, where $\ln$ is the natural logarithm function. From this plot, we select a scaling region and compute the slope of the curve in that region. This process is repeated for increasing values of the embedding dimension, $m$, and if the values of the slopes converge, then we have found the Correlation Dimension $D_{2}$ of the time-series $X(t)$. The convergence value of the slope is an estimation of the Correlation Dimension. A time-series that results from a complex non-linear dynamic system yields a larger value for the Correlation Dimension, as opposed to a time-series which results from a regular and linear dynamic system, lower Correlation Dimension values. Generally, the
Correlation Dimension, $D_{2}$, represents the independent degrees of freedom that are required for the proper description of a system or for the construction of its model.

\subsection{The Approximate Entropy}

There are various definitions of entropy, most of which usually arise from entropy computation such as the Shannon entropy or the Kolmogorov-Sinai entropy. From all known methods, Approximate Entropy ( $A p E n)$ is chosen, since it has been introduced as a quantification of regularity in data and as the natural information parameter for an approximating Markov Chain to a process (Pincus, 1991).

Given the original time-series $X(t)$, we construct a series of vectors, and then we find the heuristic estimation of an integer parameter, $m$, which in this case represents a window size. We then, one again, heuristically estimate a threshold, $r$, which arises from the product of the standard deviation of the time series and an arbitrary constant form 0 to 1 , which is kept the same for all time-series. We then apply an iterative procedure which finally produces an approximation of $A p E n(m, r)$. Generally, random time-series produce increasing values of $A p E n(m, r)$, compared to regular 


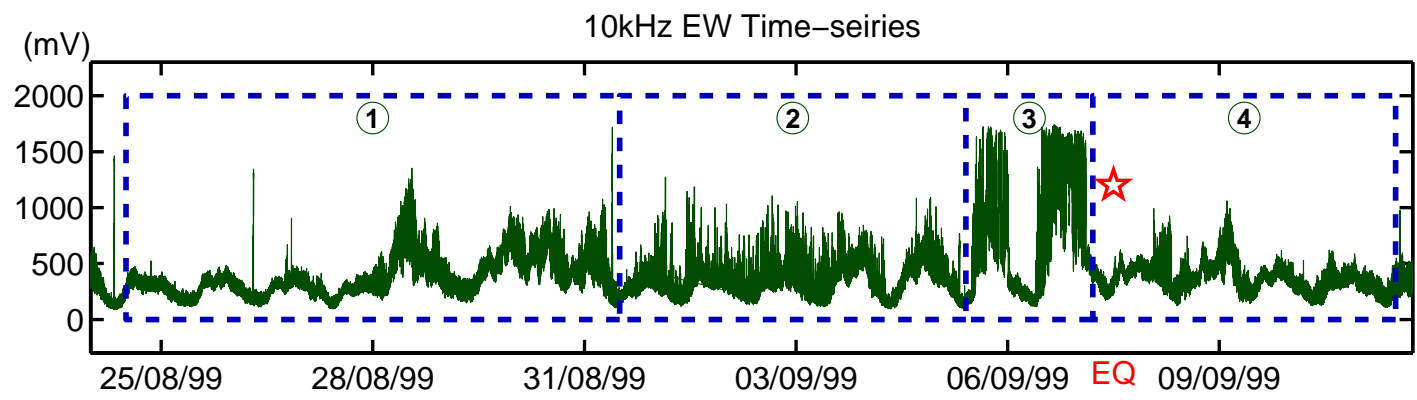

Fig. 3. View of the time-series of $10 \mathrm{kHz}$ E-W. The four epochs in which the calculation are made are depicted.

time-series, a property which we exploit here. More details as well as a more analytical description of the method are included in Appendix B.

\subsection{The fractal spectral analysis}

The concept of fractal is most often associated with irregular geometric objects that display self-similarity. Fractal forms are composed of subunits (and sub-sub-units, etc.) that resemble the structure of the overall object. The fractal analysis can be applied not just in irregular geometric forms that lack a characteristic (single) scale of length, but also to certain complex processes that generate irregular fluctuations across multiple time scales, analogous to scale-invariant objects that have a branching or wrinkly structure across multiple length scales. Earthquakes happen in self-organizing complex systems consisting of many non linear interacting units, namely opening micro-cracks. Self-organized complexity manifests itself in linkages between space and time producing fractal processes and structures. Herein, we concentrate on the question whether distinctive alterations in associated scaling parameters emerge as earthquakes are approaching.

We focus on the statistics of the detected electromagnetic fluctuations with respect to their amplitude, let's say $A\left(t_{i}\right)$. We attempt to investigate autocorrelation structures in these time-series. Any time series may exhibit a variety of autocorrelation structures; successive terms may show strong (brown noise), moderate (pink noise) or no (white noise) correlation with previous terms. The strength of these correlations provides useful information about the inherent "memory" of the system. The power spectrum, $S(f)$, which measures the relative frequency content of a signal, is probably the most commonly used technique to detect structure in time-series. If the time-series $A\left(t_{i}\right)$ is a fractal time series that series cannot have a characteristic time scale. But a fractal time series cannot have any characteristic frequency either. The only possibility is then that the power spectrum $S(f)$ has a scaling form:

$S(f) \sim f^{-\beta}$

where the power spectrum $S(f)$ quantifies the correlations at the time scale $\tau \sim 1 / f$ and $f$ is the frequency of the Fourier transform. In a $\ln S(f)-\ln (f)$ representation the power spectrum is a line with linear spectral slope $\beta$. The linear correlation coefficient, $r$, is a measure of the goodness of fit to the power law Eq. (1).

Our approach is to calculate the fractal parameter $\beta$ and the linear correlation coefficient $r$ of the power law fit dividing the signal into successive segments of 1024 samples each, in order to study not only the presence of a power law $S(f) \sim f^{-\beta}$ but, mainly, the temporal evolution of the associated parameters $\beta$ and $r$. The Continuous Wavelet Transform (CWT), using Morlet wavelet, is applied to compute the power spectrum, since being superior to the Fourier spectral analysis providing excellent decompositions within the maximum admitted window length (Kaiser, 1994).

\section{Methods - Results}

A convenient way to examine transient phenomena is to divide the measurements in time windows and analyze these windows. If this analysis yields different results for some precursory time intervals (epochs), then a transient behaviour can be extracted. We apply this technique for each of the methods used below.

We discriminate four epochs in the EM time series under study (Fig. 3). The first epoch refers to the electromagnetically quiescent period preceding the emergence of the EM anomaly. The second and third epochs include the precursory (possibly seismogenic) EM activity. We separate two time intervals during the detection of this EM anomaly, because we mainly search for the appearance of transient phenomena during the last preparation stage of the main shock. Finally, the fourth epoch refers to the period after the abrupt termination of the recorded EM anomaly.

\subsection{Application of the Delay Times method}

Through the use of the autocorrelation and mutual information functions, a value was determined for the time lag, $\tau$, that is most suitable for this study and that was $\tau=7$. The dimensions chosen for the phase space reconstruction started at $m=3$ and went to $m=20$. Both $m$ and $\tau$ values were based on the fact that after several trials these values yield the best reconstruction and thus lead to more accurate results 

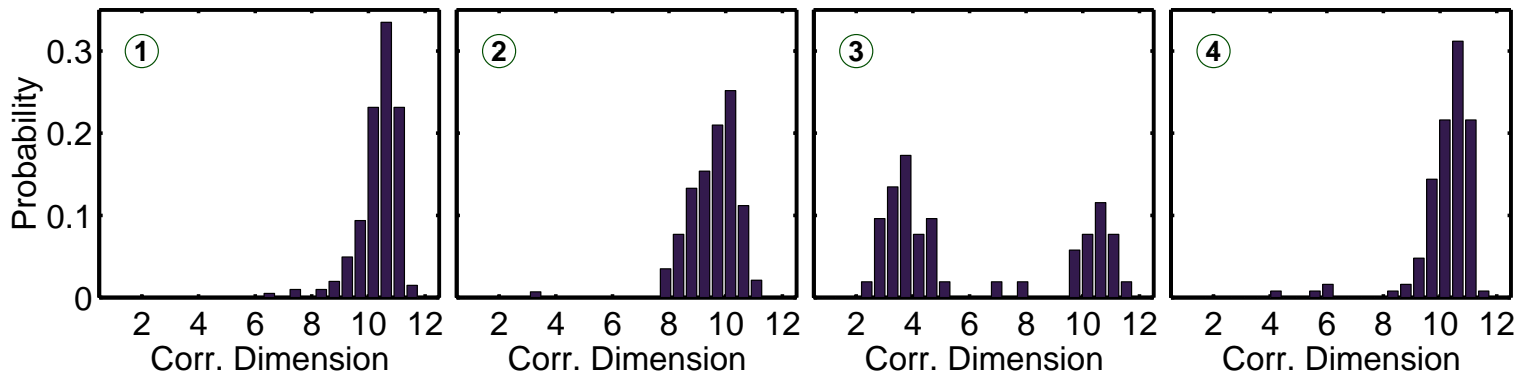

Fig. 4. We first estimate the Correlation Dimension, $D_{2}$, in consecutive segments of 3000 samples each. Then, we trace the distribution of these $D_{2}$-values for four consecutive epochs. The four epochs are depicted in Fig. 3. The epochs 1 and 4 correspond to the EM quiescence that precedes and follows respectively the EM precursory activity. The allmost similar distributions in the epochs 1 and 4 characterize the EM background (noise). In epoch 2, the little deformation of the distribution to the left side in respect to the distribution of the pure noise indicates that the initial part of the precursory emission is characterized by a little reduction of the complexity in respect to the high complexity of the pure noise. The right lobe that appears in the period 3 corresponds to the EM background, while the left lobe corresponds to the EM precursory activity. We observe a dramatic shift of the distribution of the $D_{2}$-values in epoch 3 . This evidence indicates a strong reduction of complexity during the emergence of the two strong EM bursts in the tail of the precursory emission.
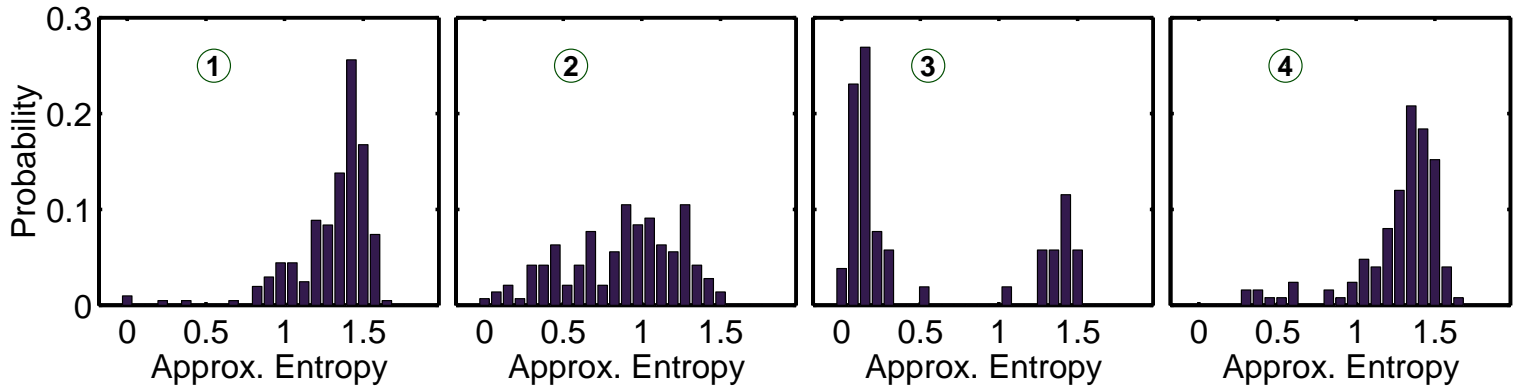

Fig. 5. We first estimate the Approximate Entropy, ApEn, in consecutive segments of 3000 samples each. Then, we trace the distribution of these $A p E n$-values for four consecutive epochs that correspond exactly to the four epochs of Fig. 4. In the epoch 2, we observe an important shift of $A p E n$-values to lower values. This indicates that the emerged EM emission has a behavior far from this of the EM background. The right lobe that appears in epoch 3 corresponds to the EM background, while the left lobe corresponds to the EM precursory activity. We observe a dramatic reduction of complexity during the emergence of the two strong EM bursts in the tail of the precursory emission.

and subject discrimination. The correlation integral was then calculated for an extended range of $r$ (up to $10^{8}$, experimentally determined).

We calculate the correlation dimension, $D_{2}$, associated with successive segments of 3000 samples each and study the distributions of correlation dimension $D_{2}$ in four consecutive time intervals (Fig. 3). We recall that the recorded VLF EM anomaly of gradual increasing activity has been launched through a long duration kilohertz EM quiescence, while they ceased a few hours before the Athens earthquake. The first time interval corresponds to the quiescence EM period preceding the EM anomaly. The second and third time intervals correspond to the period of the recorded precursory anomaly; the third time interval includes the two strong impulsive bursts in the tail of the precursory emission. The fourth time interval refers to the quiescence period after the cessation of the precursory emission.

We underline the similarity of the distributions of the $D_{2}$ values in the first and fourth time intervals (Fig. 4). This allmost common distribution characterizes the order of com- plexity in the background noise of the EM time series. The associated predominance $D_{2}$-values, from 7 up to 10 , indicate a strong complexity and non linearity. Notice, that a relevant lobe remains in the distributions of $D_{2}$-values in the second and third time interval, as it was expected.

Now, we focus on the second and third time intervals, namely during the emergence of the precursory emission. We observe a significant decrease of the $D_{2}$-values as we move from the second to the third time window. The observed significant decrease of the $D_{2}$-values signals a strong loss of complexity in the underlying fracto-electromagnetic mechanism during the launching of the two strong EM bursts in the tail of the precursory emission. This evidence might be indicated by the appearance of a new phase in the tail of the earthquake preparation process, which is characterized by a higher order of organization. Sufficient experimental evidence seems to support the association of the aforementioned two EM bursts with the nucleation phase of the impending earthquake (Eftaxias et al., 2001; Kapiris et al., 2004b). 

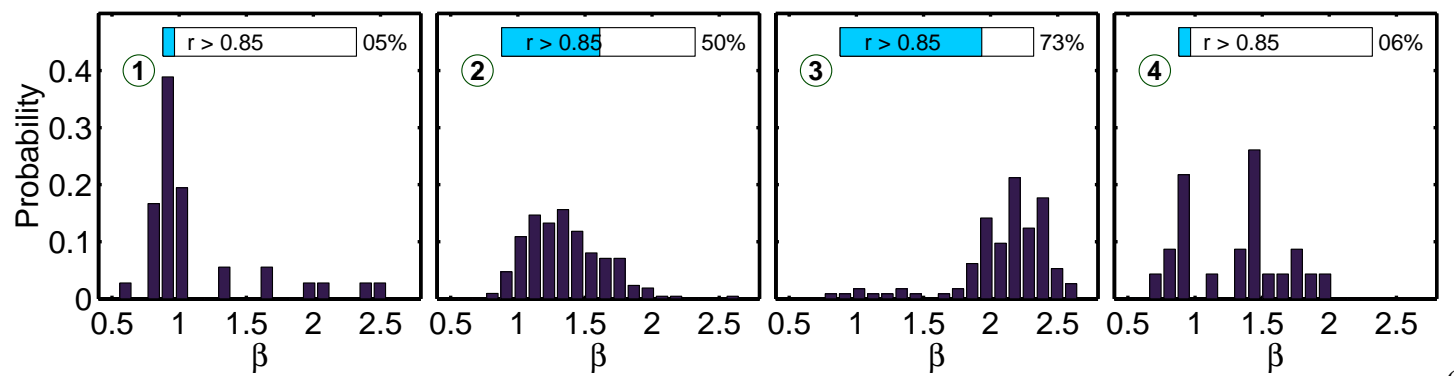

(a)
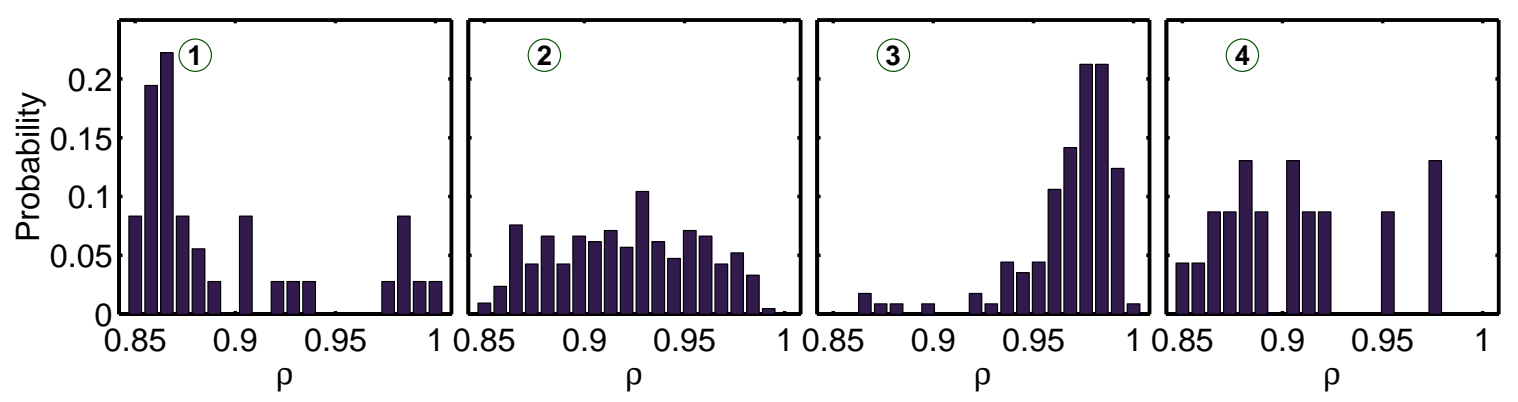

(b)

Fig. 6. (a) We first estimate the exponent $\beta$, in consecutive segments of 1024 samples each. Then, we trace the distribution of these $\beta$-values for four consecutive epochs. The four epochs are depicted in Fig. 3 and correspond exactly to the four epochs of Fig. 4 and Fig. 5 . Insets show percentage of segments with $r>0.85$. It is evident that the closer the final stage of seismic process, the larger the percentage of segments with $r>0.85$, and the larger shift of $\beta$ to higher values. Notice that in epoch 3 the signal becomes persistent. (b) The propability distributions of linear coef. $r$ beyond 0.85 .

These findings suggest that there is important information in terms of correlation dimension hidden in the heterogeneities of the pre-seismic time series. The correlation dimension $D_{2}$ in the sequence of the precursory EM pulses seems to measure the distance from the global instability: the larger the $D_{2}$-values the larger the distance from the critical point.

\subsection{Application of the Approximate Entropy method}

The Approximate Entropy was computed for a variety of $r$ values proposed by previous researchers and it was found that the optimum value yielding clearest discrimination was the value $r=0.65 S T D$, where $S T D$ is the standard deviation of the time-series.

We calculate the Approximate Entropy associated with successive segments of 3000 samples each and study the distributions of the ApEn-values in four consecutive time intervals, as in the case of the study in terms of Correlation Dimension (Fig. 5).

We observe again the similarity of the distributions of the $A p E n$-values in the first and fourth time intervals: this allmost common distribution refers to the background noise of the EM time series. A relevant lobe remains in the distributions of the $A p E n$-values in the second and third time inter$\mathrm{val}$, as it was expected.

Now, we concentrate on the second and third time intervals, namely during the emergence of the precursory emission. We observe a significant decrease of the $A p E n$-values as we move from the second to the third time window. The observed considerable decrease of the ApEn-values in the third time interval reveals a strong loss of complexity in the underlying mechano-electromagnetic transduction during the launching of the two strong EM bursts in the tail of the precursory emission. In other words, the pre-focal area seems to be less responsive to the external stimuli when the pre-seismic EM signals are characterized by low ApEnvalues.

In summary, in the pre-seismic EM time-series the values of the Correlation Dimension and Approximate Entropy are reduced as the main event is approached. This evidence indicates that the underlying fracto-electromagnetic mechanism exhibits a strong complexity and non-linearity far from the global failure. A significant loss of complexity and non-linearity is observed close to the global instability. This considerable alteration in both $D_{2}$-values and $A p E n$-values might be considered as candidate precursor of the impending event.

\section{Remark}

According to the appendixes, the method of Correlation Dimension (CorrDim) embeds the original time series into a phase space of dimension 3 to 20, examining thus the probability distribution of a norm defined in this phase space, contrary to the Approximate Entropy method which embeds the original time series in a 2-dimensional phase space only. As a result, the CorrDim method yields a more detailed description of a system's complexity, comparing to 
the ApEn method, which focuses mainly to coarse grained characteristics. When the examined time series is generated by a low dimensional process, it is better to use the $A p E n$ method. On the other hand, when the complexity of the examined time series increases, the CorrDim method is more suitable as it is more sensitive to high complexity. In the cases of epochs 2 and 3 in Figs. 4 and 5, we are able to observe the above mentioned property. Focusing to epoch 2 in Fig. 5, the shift of ApEn-values to lower values witnesses the reduction of complexity, and thus the emergence of the precursor. In epoch 3 the complexity has further been diminished. Thus the ApEn method is the proper one to describe the associated grouping activity of the structures. Indeed, we observe that the probability distributions of EQ- $D_{2}$ and Background- $D_{2}$ values seem to be similar (epoch 3 in Fig. 4), while in the case of $A p E n$ method, the probability of EQ-ApEn values is larger than the Background ApEn-values.

\subsection{Application of fractal-dynamics}

The spectral fractal analysis reveals that the pre-seismic electromagnetic fluctuations exhibit hidden scaling structure. We observe alterations in the associated dynamical parameters, which seem to uncover important features of the underlying earthquake preparation process (Kapiris et al., 2002; Eftaxias et al., 2003; Kapiris et al., 2003, 2004b,a; Eftaxias et al., 2004).

Figure 6 exhibits the temporal evolution of $r$ as the main event is approached. We observe a gradual increase of the correlation coefficient with time: at the tail of the precursory activity the fit to the power law is excellent. The fact that the data are well fitted by the power-law (1) suggests that the pre-seismic EM activity could be ascribed to a multi-timescale cooperative activity of numerous activated fundamental units, namely, emitting-cracks, in which an individual unit's behavior is dominated by its neighbours so that all units simultaneously alter their behavior to a common large scale fractal pattern. On the other hand, the gradual increase of $r$ indicates that the clustering in more compact fractal structures of activated cracks is strengthened with time.

Now we focus on the behavior of $\beta$-exponent. Two classes of signal have been widely used to model stochastic fractal time series (Heneghan and McDarby, 2000): fractional Gaussian noise (fGn) and fractional Brownian motion (fBm). These are, respectively, generalizations of white Gaussian noise and Brownian motion. The nature of fractal behavior (i.e. fGn versus $\mathrm{fBm}$ ) provides insight into the physical mechanism that generates the correlations: the $\mathrm{fBm}$ represents cumulative summation or integration of a fGn. A formal mathematical definition of continuous $\mathrm{fBm}$ was first offered by (Mandelbrot and Ness, 1968).

For the case of the $\mathrm{fBm}$ model the scaling exponent $\beta$ lies between 1 and 3 , while, the range of $\beta$ from -1 to 1 indicates the regime of fGn (Heneghan and McDarby, 2000). Fig. 6 reveals that during the epochs 2 and 3 (Fig. 3) the $\beta$-values are distributed in the region from 1 to 3 . This means that the possible seismogenic EM activity follows the fBm model.

We concentrate on the quiescent EM period (first epoch in Fig. 3), preceding the emergence of the EM anomaly (second and third epochs in Fig. 3). We observe that only a very small number of segments, approximately $5 \%$, follows the power law (1) (see inserts in Fig. 6). We can conclude that during the epoch 1 the associated time series do not behave as a temporal fractal. Moreover, if we concentrate on the 5\% of the segments, the associated $\beta$-values range from 0 to 1 , namely, this minority of segments may follow the fGn model. We conclude that regime of the quiescent period is quite different from those of the possible seismogenic emission. The transition to the fractal structure and $\mathrm{fBm}$ class further identify the launch of the fracto-electromagnetic emission from the background (noise) of EM activity.

The distribution of $\beta$-exponents is also shifted to higher values (Fig. 6) during the precursory period.

The precursory shift of the distribution of both $\beta$-exponent and $r$-coefficient to higher values reveals important features of the underlying mechanism. The fractal-laws observed corroborate to the existence of memory; the system refers to its history in order to define its future. As the $\beta$-exponent increases the spatial correlation in the time-series also increases. This behaviour signals the gradual increase of the memory, and thus the gradual loss of complexity in the process. Maslov et al. (1994) have formally established the relationship between spatial fractal behaviour and long-range temporal correlations for a broad range of critical phenomena. By studying the time correlations in the local activity, they show that the temporal and spatial activity can be described as different cuts in the same underlying fractal. Laboratory results support this hypothesis: Ponomarev et al. (1997) have reported in phase changes of the temporal and spatial Hurst exponents during sample deformation in laboratory acoustic emission experiments. Consequently, the observed increase of the temporal correlation in the pre-seismic time-series may also reveal that the opening-cracks are correlated at larger scale length with time.

The following feature goes to the heart of the problem: first, single isolated micro-cracks emerge which, subsequently, grow and multiply. This leads to cooperative effects. Finally, the main shock forms. The challenge is to determine the "critical time-window" during which the "short-range" correlations evolve into "long-range" ones. Fig. 6a indicates that the closer the global instability the larger the percentages of segments with $r$ close to 1 and the larger the shift of $\beta$-exponent to higher values; the $\beta$-values are maximal at the tail of the pre-seismic state (Fig 6b). This behaviour may reveal the "critical time-window".

The exponent $\beta$ is related to the Hurst parameter, $H$, by the formula (Turcotte, 1992)

$\beta=2 H+1$ with $0<H<1$ and $1<\beta<3$

for the fBm model (Mandelbrot and Ness, 1968; Heneghan and McDarby, 2000). Consequently, segments with Hurst- 
exponents estimated by the previous formula out of the range $0<H<1$ do not follow the fBm model.

The exponent $H$ characterizes the persistent/antipersistent properties of the signal according to the following scheme. The range $0.5<H<1 \quad(2<\beta<3)$ suggests persistence of the signal, i.e. if the amplitude of fluctuations increases in a time interval it is likely to continue increasing in the interval immediately following. The range $0<H<0.5$ $(1<\beta<2)$ suggests anti-persistence of the signal, i.e. if the fluctuations increase in a period, it is likely to continue decreasing in the interval immediately following and vice versa. $H=0.5(\beta=2)$ indicates no correlation between the process increments, that is the system is characterized by random fluctuations (Mandelbrot and Ness, 1968; Hristopulos, 2003). Consequently, the particular value $\beta=2$ takes on a special physical meaning: it signals the transition from anti-persistent to persistent behaviour in the time series.

\section{Remark}

As it was mentioned, the range $-1<\beta<1$ implies fGn behavior. For this model the exponent $\beta$ is related to the Hurst parameter by the formula $\beta=2 H-1$ (Heneghan and McDarby, 2000). Thus, $H$ is also constrained to lie between 0 and 1 in epochs 1 and 4 (Fig. 6).

Physically, the Hurst exponent express the strength of the effect of excitation associated with the preceding event on succeeding events, or equivalently, the degree of negative/positive feedback in the dynamics. The range $0<H<0.5$ $(1<\beta<2)$ during the first period of the EM precursor indicates a very large anti-persistency. This behaviour implies a set of fluctuations tending to induce a greater stability in the system. The observed shift of local $H$-exponents can be understood if we accept that the micro-heterogeneity of the system becomes less anti-correlated with time. The anti-persistent properties during this period are consistent with the existence of a non-linear feedback mechanism that "kicks" the cracking rate in the pre-focal area away from extremes. The systematic increase of the $\beta$-exponent (or Hurstexponent) indicates that the fluctuations become less anticorrelated with time, i.e. the nonlinear negative feedbacks gradually lose their ability to kick the system away from extremes. In other words, the decrease of heterogeneity appears to lead to a decrease in the ability to drive the system away from a persistent mode of opening-cracks evolution. It might be argued that "the first anti-persistent part of the precursory electromagnetic radiation is triggered by micro-fractures in the disordered system that surrounds the, allmost, homogeneous backbones within the pre-focal area".

For times close to breakthrough, allmost homogeneous backbones of high strength sustain the elastic strain energy. In the limit of a homogeneous system, once a crack nucleates in the rock, the stress is enhanced at its tip and therefore the next micro-crack almost surely develops at the tip. The appearance of persistence properties within the two strong impulsive signals at the tail of the precursory time series is thought to be clue to the fracture of the high strength ho- mogeneous backbones (Kapiris et al., 2004b; Eftaxias et al., 2004; Kapiris et al., 2004a). This behaviour may witness that the system has been starting to self-organize by a positive feedback process, and thus, this acquires to a great degree the property of irreversibility. The concept that the launch of the persistence activity could give a significant hint of a considerable probability for a forthcoming global instability, namely, a significant event, can be accepted.

We have paid attention to the following experimental evidence: The accelerating EM precursor (Fig. 3) ends in two clear persistent signals with an energy ratio (second to first signal) $\sim 5$. The radar interferometry analysis showed activation of two separate faults with corresponding energy release ratio (second to first signal) $\sim 5$ (Eftaxias et al., 2001; Kapiris et al., 2004a). This surprising correlation in the energy domain enhances the consideration that the launch of persistent dynamics may signals the emergence of the nucleation stage of earthquake preparation.

It is worth mentioning that laboratory experiments by means of acoustic and electromagnetic emission also show that the main rupture occurs after the appearance of persistence behaviour (Ponomarev et al., 1997; Alexeev and Egorov, 1993; Alexeev et al., 1993) in the time-series.

We conclude that the aforementioned three methods present coherent results: they clearly and accurately identify significant alteration in terms of $D_{2}$-values, $A p E n$-values, and Hurst-values, between the initial and terminal phase of the pre-seismic EM emission. The coherent results signal a significant loss of complexity in the tail of the precursory EM activity, while the underlying fracto-electromagnetic mechanism becomes persistent. The dynamical parameters, $D_{2}, A p E n$, and $H$, seems to represent a measure of the distance of the system from the global instability. The lower the $D_{2}$-values, the lower the $A p E n$-values, and the higher the Hurst-values, then, the smaller the distance of the system from the "critical point" (global instability).

\section{Remark}

A fundamental characteristic of probable EM precursors is their appearance in a wide frequency band, ranging from DC-ULF, ELF, VLF, and LF to VHF. The time elapsed from the detection of the DC-ULF pre-seismic EM activity to the occurrence of strong EQ (Varotsos et al., 1996; Hayakawa et al., 1999, 2000; Telesca and Lapenna, 2001; Varotsos and Sarlis, 2002; Ramirez-Rojas et al., 2004), is longer than the time for the VLF-VHF emissions e.g. (Gershenzon and Bambakidis, 2001; Eftaxias et al., 2001, 2002). Therefore, we can accept the concept that pre-seismic signals may arise from different mechanisms, which do not lie within the same time scales.

Authors have studied pre-seismic ULF geo-magnetic (Hayakawa et al., 1999, 2000) and geo-electrical signals (Telesca and Lapenna, 2001) in terms of fractal spectral analysis. Regarding the behavior of the spectral $\beta$-exponent observed, it seems that this is in contrast to the behavior observed during the present study. Characteristically, the 
$\beta$-values showed a tendency to gradually decrease during the process of the earthquake preparation. We think that this difference supports the hypothesis that the ULF signals on one hand and the VLF-VHF signals on the other hand may have originated on different mechanisms. Indeed, it has been suggested that the ULF geo-electical signals could be explained in these terms: (i) "Pressure Stimulated Currents" that are transient currents emitted from a solid containing electric dipole upon a gradual variation of pressure (Varotsos and Alexopoulos, 1984a,b; Varotsos et al., 1996). (ii) The electro-kinetic effect e.g (Mizutani and Ishido, 1976; Dobrovolsky et al., 1989; Gershenzon and Bambakidis, 2001). Because electro-kinetic effect is controlled by the diffusion of water with the diffusion time comparable to the period of ULF emissions, more energy is provided to the ULF range (Gershenzon and Bambakidis, 2001). We note that recently, (Surkov et al., 2002) have explained the logarithmic dependence of electric field amplitude $E$ on the earthquake magnitude $M$ that is indicated by experimental results (Varotsos et al., 1996).

\section{From the normal state to the seismic shock or epilep- tic seizure in terms of complexity}

The world is made of highly interconnected parts on many scales, the interactions of which results in a complex behavior that requires separate interpretation of each level. The laws that describe the behavior of a complex system are qualitatively different from those that govern its units. New features emerge as one moves from one scale to another, so it follows that the science of complexity is about revealing the principles that govern the ways in which these new properties appear.

A basic reason for our interest in complexity is the striking similarity in behaviour close to irreversible phase transitions among systems that are otherwise quite different in nature (Stanley, 1999, 2000; Sornette, 2002; Vicsek, 2001, 2002; Turcotte and Rudle, 2002). Recent studies have demonstrated that a large variety of complex processes, including earthquakes (Bak and Tang, 1989; Bak, 1997), forest fires (Malamud et al., 1998), heartbeats (Peng et al., 1995), human coordination (Gilden et al., 1995), neuronal dynamics (Worrell et al., 2002), financial markets (Mantegna and Stanley, 1995) exhibits statistical similarities, most commonly powerlaw scaling behaviour of a particular observable. Stanley (2000) offer a brief and somewhat parochial overview of some "exotic" statistical physics puzzles of possible interest to biophysicists, medical physicists, and econophysics.

Interestingly, authors have suggested that earthquake's dynamics and neurodynamics could be analyzed within similar mathematical frameworks (Rundle et al., 2002). Characteristically, slider block models are simple examples of driven non-equilibrium threshold systems on a lattice. It has been noted that these models, in addition to simulating the aspects of earthquakes and frictional sliding, may also represent the dynamics of neurological networks (Rundle et al.,
1995, and references therein). A few years ago, Bak et al. (1987) coined the term self-organized criticality (SOC) to describe the phenomenon observed in a particular automaton model, nowadays known as the sandpile-model. This system is critical in analogy with classical equilibrium critical phenomena, where neither characteristic time nor length scales exist. In general, the strong analogies between the dynamics of the "self-organized-criticality" (SOC) model for earthquakes and that of neurobiology have been realized by numerous of authors (Hopfield, 1994), (Herz and Hopfield, 1995, and references there in), (Usher et al., 1995), (Zhao and Chen, 2002, and references there in), (Beggs and Plenz, 2003).

Complexity does not have a strict definition, but a lot of work on complexity centers around statistical power laws, which describe the scaling properties of fractal processes and structures that are common among systems that at least qualitative are considered complex. The big question is whether there is a unified theory for the ways in which elements of a system organize themselves to produce a behavior that is followed by a large class of systems (Vicsek, 2002).

The aforementioned concepts motivated us to investigate whether common precursory patterns are emerged during the precursory stage of both epileptic seizure and earthquake (Li et al., 2004).

The brain possesses more than billions neurons and neuronal connections that generate complex patterns of behaviour. Electroencephalogram (EEG) provides a window, through which the dynamics of epilepsy preparation can be investigated. Fig. 7 exhibits rat epileptic seizure.

As in the case of the pre-seismic EM emission, we monitor the evolution of fractal characteristics of pre-epileptic activities toward criticality in consecutive time windows. Our analysis reveals that numerous distinguishing features were emerged during the transition from normal states to epileptic seizures (Li et al., 2004): (i) appearance of long range power-law correlations, i.e. strong memory effects; (ii) increase of the spatial correlation in the time-series with time; (iii) gradual enhancement of lower frequency fluctuations, which indicates that the electric events interact and coalesce to form larger fractal structures; (iv) decrease of the fractal dimension of the time series; (v) decrease with time of the anti-persistent behavior in the precursory electric time series; (vi) appearance of persistent properties in the tail of the preepileptic period. Fig. 7 shows the aforementioned precursory behavior.

Notice that the aforementioned candidate precursors of the impending epileptic seizure or earthquake are launched in a way striking similar to those occurring just before the "critical point" of phase transition in statistical physics. Based on this similarity, it might be argued that the earthquake/epilepsy may be also viewed as "a generalized kind of phase transition" (Kapiris et al., 2004b; Contoyiannis et al., 2004).

Our results indicate that an individual firing neuron or an opening crack is dominated by its neighbours so that all activated biological or geological units simultaneously alter their 
$(\mathrm{mV})$

EEG Time-seiries
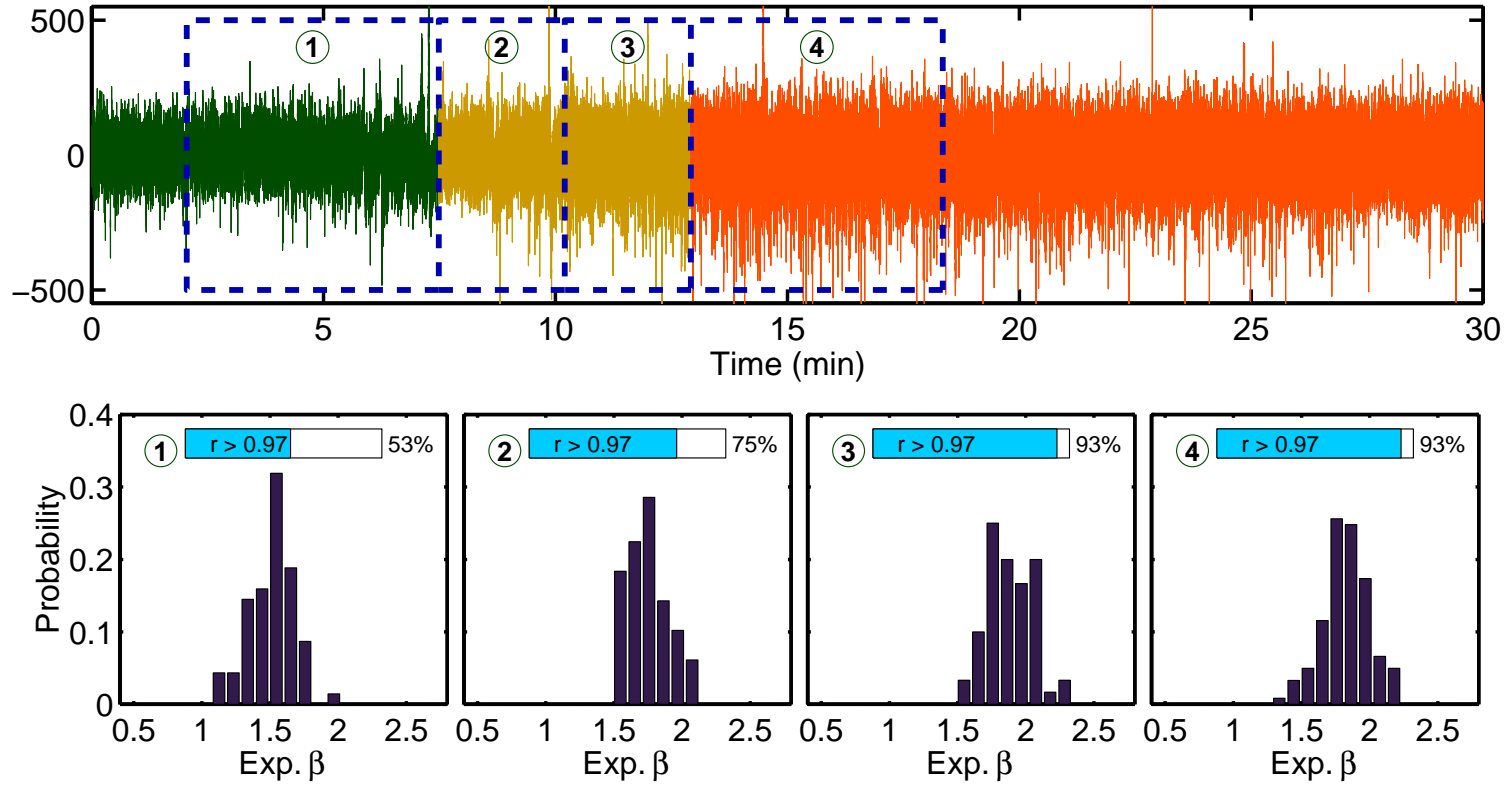

Fig. 7. A rat epileptic seizure (red signal) in EEG time-series (upper part). Two electrodes were placed in epidural space to record the EEG signals from temporal lobe. EEG signals were recorded using an amplifier with band-pass filter setting of $0.5-100 \mathrm{~Hz}$. The sampling rate was $200 \mathrm{~Hz}$. Bicuculline i.p injection was used to induce the rat epileptic seizure. The injection time is at 7:49 (m-s) and the seizure at 12:55 (m-s), respectively. This pre-seizure period is depicted by the yellow part of the EEG time series. We estimate the exponent $\beta$, in consecutive segments of 1024 samples each. Then, we trace the distribution of these $\beta$-values for four consecutive epochs (lower part). The four epochs are depicted in the upper part with numbered dashed frames. Insets show percentage of segments with $r>0.97$. Notice that at the last stage of the pre-ictal period (epoch 3) the signal emerges persistent behavior.

behavior to a common fractal pattern as the epileptic seizure or the earthquake is approaching. Interestingly, common alterations in the associated parameters are emerged indicating the approach to the global instability in harmony with relevant theoretical suggestions (Hopfield, 1994; Herz and Hopfield, 1995; Usher et al., 1995; Zhao and Chen, 2002; Rundle et al., 2002; Beggs and Plenz, 2003). Consequently, the present analysis seems to support the concept that, indeed, a unified theory may describe the ways in which elements of a biological or geological system organize themselves to produce a catastrophic event.

\section{From the normal state to the seismic shock or heart- failure in terms of Correlation Dimension, Approxi- mate Entropy and Multifractality}

Recently, Fukuda et al. (2003) have investigated similarities between communication dynamics in the Internet and the autonomic nervous system. They found quantitative similarities between the statistical properties of (i) healthy heart rate variability and non-congested Internet traffic, and (ii) diseased heart rate variability and congested Internet traffic. The authors conclude that their finding suggest that the understanding of the mechanisms underlying the "human-made" Internet could help to understand the "natural" network that controls the heart. In the sense of this approach, we search for similarities from the normal state to the seismic shock or heart-failure.

\subsection{Similarities in terms of multifractality}

Mathematical analysis of both long-term heart-rate fluctuations (Ivanov et al., 1999; Stanley et al., 1999; Goldberger et al., 2002) and pre-seismic EM emissions (Kapiris et al., 2004b) show that they are members of a special class of complex processes, termed multi-fractals, which require a large number of exponents to characterize their scaling properties. In general, the detection of multi-fractal scaling may indicate that the underlying nonlinear mechanism regulating the system might interact as part as a coupled cascade of feedback loops in a system operating far from equilibrium (Meneveau and Sreenivasan, 1987).

Monofractal signals can be indexed by a single global exponent, i.e. the Hurst exponent $H$ (Hurst, 1951). Multifractal signals, on the other hand, can be decomposed into many subsets characterized by different local Hurst exponents $h$, which quantify the local singular behavior and thus relate to the local scaling to the time series. Thus, multifractal signals require many exponents to characterize their scaling properties fully (Vicsek, 1993). The statistical properties of the different subsets characterized by these different exponents $h$ can be quantified by the function $D(h)$, where $D\left(h_{0}\right)$ is the fractal dimension of the subset of the time series 

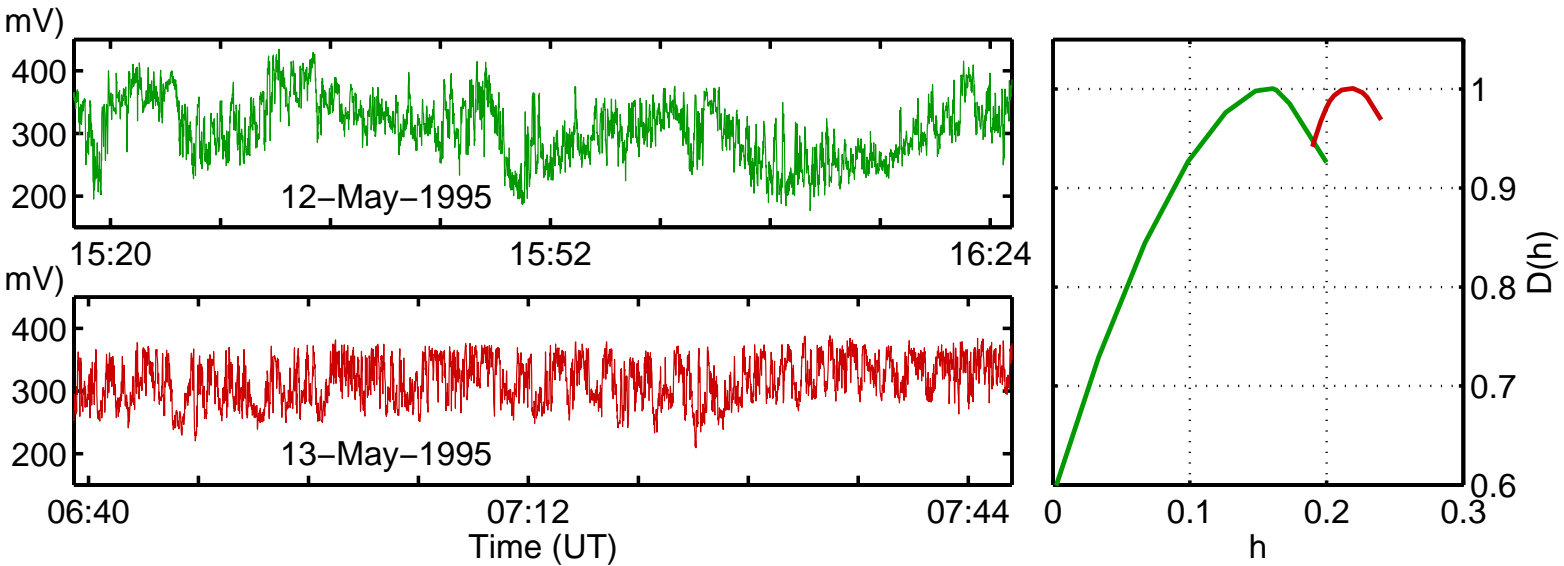

Fig. 8. Two segments of the precursory $41 \mathrm{MHz}$ electromagnetic signal, recorded on 12 May 1995 (upper row) and 13 May 1995 (lower row) before the Kozani-Grevena earthquake $M s=6.6$ on May 13, 1995 at 08:47:12.9 UTC. On the right part of the figure the corresponding fractal dimensions $D(h)$ are presented.

characterized by the local Hurst exponent $h_{0}$. Ivanov et al. (1999) have uncovered a loss of multifractality, as well as a loss of the anti-persistent behaviour, for a life-threatening condition, congestive heart failure. Following the method of multifractal analysis used by Ivanov et al. (1999), we examined multi-fractal properties in the VHF time series, namely, the spectrum of the fractal dimension $D(h)$, as a candidate precursor of the Kozani-Grevena earthquake (Kapiris et al., 2004b). Figure 8 shows that as the main event approaches, the EM time series manifest: a significant loss of multifractal complexity and reduction of non-linearities, displaying a narrow (red) multifractal spectrum, and their fluctuations become less anti-correlated, as the dominant local Hurst exponents is shifted to higher values. These results reflect that for both the heart and pre-focal area at high risk the multi-fractal organization allmost breaks down.

In summary, the multifractality of the heart-beat time series and pre-seismic EM time series further enables us to quantify the greater complexity of the "healthy" dynamics compared to those of "pathological" conditions in both heart and pre-focal area.

\subsection{Similarities in terms of Correlation Dimension and Ap- proximate entropy}

Recently, we have studied several methods which have been used for the categorization of two subjects groups, one which represents subjects with no prior occurrence of coronary disease events and another group who have had a coronary disease event (Nikolopoulos et al., 2003; Karamanos et al., 2004). It is worth mentioning that the Delay Times method and the computation of the Approximate Entropy present coherent results and succeed in clearly and accurately differentiating healthy subject ECGs from those of unhealthy subjects and coronary patients.

Heart Rate Variability (HRV) time series coming from coronary patients exhibit more regular and periodical be- haviour compared to ones coming from healthy subjects. The correlation dimensions of healthy time series are about $D_{2} \approx$ 9 when the respective ones for the patients are about $D_{2} \approx 6$ (Nikolopoulos et al., 2003). Similarly, the mean $A p E n$ value for the healthy time series was about $A p E n \approx 1.2$ and for the patients $A p E n \approx 0.4$ (Nikolopoulos et al., 2003). A similar reduction of complexity for heart failures has been observed in terms of Block-Entropy by some of the present authors (Karamanos et al., 2004). It is important to note that the $D_{2}$-values and $A p E n$-values associated with the second time interval of the pre-seismic EM time series are close to the ones coming from healthy subjects, while, the $D_{2}$-values and $A p E n$-values associated with the third time interval are close to the ones coming from patient subjects. Based on this analogy, we could say that the EM emissions in second and third time interval implies a kind of "healthy" and "patient" pre-focal area correspondingly. We focus on this analogy.

We recall that the EM time series in the second time interval is characterized by strong anti-persistence and multifractality. The multifractality indicates that the underlying nonlinear mechanism regulating the system might interact as part as a coupled cascade of feedback loops in a system operating far from equilibrium (Meneveau and Sreenivasan, 1987). The anti-persistent properties during this period imply a set of fluctuations tending to induce a greater stability in the system. Thus, by the term "healthy pre-focal area", we mean a candidate focal area, which is consistent with a non-linear negative feedback system that "kicks" the cracking rate away from extremes.

By the term "patient pre-focal area", we mean a pre-focal area in which the system has been starting to self-organize by a non-linear positive feedback process, and thus, this acquires to a great degree the property of irreversibility. This behaviour may imply that the nucleation stage, the most interesting phase in the preparation process of the catastrophic fracture, has already been emerged. 
The study of pre-failure EM signals seems to provide a way for observing the Earth's crust ability to respond to stresses. Hallmarks of the "patient pre-focal area" are: the persistence behavior, the low multifractality, the low Correlation Dimension and the low Approximate Entropy. These hallmarks characterize a life-threatening condition for the human heart, too. Briefly, the "patient pre-focal area" and the "patient human heart" are characterized by a low complexity. On the other hand, signatures indicating the "healthy pre-focal area" are: the anti-persistence behavior, the high multifractality, the high Correlation Dimension, and the high Approximate Entropy. These signatures also characterize healthy human heartbeat. Briefly, the "healthy pre-focal area" and the healthy human heart are characterized by high complexity.

In a geometrical sense, the dynamical parameter $\beta$ specifies the strength of the signal's irregularity as well. Qualitatively speaking, the irregularity of the signal decreases as the memory in the time-series increases. For the $\mathrm{fBm}$ model the fractal dimension $d$ is found from the relation $d=(5-\beta) / 2$, which, after considering the aforementioned shift of $\beta$ exponent to higher values, leads to a decrease of fractal dimension as the earthquake approaches. We recall the WestGoldberger hypothesis that a decrease in healthy variability of a physiological system is manifest in a decreasing fractal dimension (Goldberger et al., 2002, and references there in). Our results imply that this hypothesis could be extended to geological systems as well.

\section{Conclusions}

A method to asses the approach to the global instability has been applied in EM pre-seismic anomalies. The study of these pre-failure signals seems to provide a way for observing the Earth's crust ability to respond to stresses. The Delay Times method, the computation of the Approximate Entropy, and the monitoring of alteration of Fractal Spectral characteristics of pre-seismic EM activity toward global instability in consecutive time windows, present coherent results and succeed in a potential differentiation of the nucleation phase from previous stages of the earthquake preparation process. More precisely, the emergence of long-range correlations, i.e. appearance of long memory effects, the increase of the spatial correlation in the time series with time, the predominance of large events with time, as well as the gradual decrease of the anti-persistent behaviour may indicate the approach to the nucleation phase of the impending catastrophic event. The appearance of persistent properties in the tail of the precursory time series, the significant divergence of the energy release, the sharp significant decrease of the Approximate Entropy, and the quick reduction of the Correlation Dimension as well, all these, may hints that a new phase, probably the nucleation phase of the earthquake, has been started. This analysis may provide a useful way to the understanding of the fracture in the disordered media. The agreement of the "diagnostic" information given by each one of the meth- ods indicates the necessity of further investigation, combined use, and complementary application of different approaches.

The performed analysis reveals that common precursory signs emerge in terms of fractal dynamics as the epileptic seizure and earthquake are approaching: common distinctive alterations in associated scaling dynamical parameters emerge as biomedical or geophysical shock is approaching. The experimental results verify relevant theoretical suggestions that earthquake dynamics and neural seizure dynamics should have many similar features and should be analyzed within similar mathematical frameworks (Hopfield, 1994; Herz and Hopfield, 1995; Usher et al., 1995; Zhao and Chen, 2002; Rundle et al., 2002; Beggs and Plenz, 2003).

In principle, it is difficult to prove associations between events separated in time, such as EQs and their precursors. The present state of research in this area requires a refined a definition of a possible pre-seismic anomaly in the record of EM radiation, and also the development of more objective methods of distinguishing seismogenic emissions from non-seismic EM events. A study in terms of complexity would seem to be useful in this regard. EEG time-series provide a window through which the dynamics of biological shock preparation can be investigated in the absence of non-biological events. We observe that both kinds of catastrophic events under investigation follow common behavior in their pre-catastrophic stage. This evidence may support the seismogenic origin of the detected EM anomaly.

We find also quantitative similarities between the properties of (i) healthy heart rate variability and initial antipersistence part of the pre-seismic EM time series, and (ii) diseased heart rate variability and terminal persistence part of the pre-seismic EM activity. These similarities have been emerged in terms of Correlation Dimension, Approximate entropy, and multifractal dynamics.

Fukuda et al. (2003) recall that very simple models of very complex systems in many cases provide deep insights. For example the Ising model and its simple variants as the Heisenberg model are sufficient to quantitatively describe a wealth of very complex systems in regions of their respective phase diagrams where scale invariant is displayed. The principle of "universality" in chemistry and physics, whereby diverse systems are described by the identical (simple) model, may have its counterpart in physiology (Stanley, 1999). Even the numerical values of the critical-point exponents describing the quantitative nature of the singularities are identical for large groups of apparently diverse physical systems. It was found empirically that one could form an analog of the Mendeleev table if one partitions all critical systems into "universality classes". Two systems with the same values of critical-point exponents and scaling functions are said to belong to the same universality class. In the frame of this approach we have shown that the pre-seismic VHF emission belongs to the 3D-Ising-transition class (Contoyiannis et al., 2004). Fukuda et al. (2003) argue that their finding suggest that the understanding of the mechanism underlying the "human-made" internet could help to understand the "natural" network that controls the heart. In this sense, it 
appears that the fracture in the disordered systems may provide another useful "model system" to investigate the mechanism responsible for the dynamics of the autonomic nervous system (ANS), which controls involuntary the heart or the epilepsy generation. In terms of complexity, this possibility is not implausible.

The science of complexity is in its infancy, and some research directions that today seem fruitful might eventually prove to be academic cul-de-sacs. Sethna et al. (2001) show that the seemingly random, impulsive events by which many physical systems evolve exhibit universal, and, to some extend, predictable behavior. Nevertheless, it is reasonable to believe that the results of the present study indicate that it is useful to transfer knowledge from the domain of biomedical shock preparation to the domain of earthquake generation and vice versa. This work could serve as an invitation to other specialists in these areas to transfer knowledge from the one field of research to the other.

\section{Appendix A Delay Times method}

Given a timeseries, $X(t), t$ is an integer, $t \in(1, N)$ and $N$ is the total number of timeseries points. The Delay Times method was first established by Grassberger and Procaccia (1983) and based on the Takens Theorem (Takens, 1981). According to this method, the timeseries $x(t)$ is a measure of a single coordinate of an $m$-dimensional system's underlying dynamics. Assuming $\mathrm{m}$ is the embedding dimension (the dimension of space in which the assumed system's trajectory is unfolded) and $\tau$ is the time lag, then phase space reconstruction (described below) is performed with time delays and the following $m$-dimensional vectors are constructed:

$\boldsymbol{x}(t)=[X(t), X(t-r), X(t-2 \tau), \ldots, X(t-(m-1) \tau)](\mathrm{A} 1)$

In this way, using the original timeseries, $X(t)$, we are able to construct a new vector timeseries, $\boldsymbol{x}(t)$, which represents the trajectory from $\boldsymbol{x}(0)$ up to and including $\boldsymbol{x}(t)$ within the reconstructed phase space.

These vectors are defined in an $m$-dimensional phase space and are used in constructing the trajectory of the signal dynamics to this space. If the original phase space of the dynamics produce the attractor $A$, then the reconstruction of the phase space with the Delay Times method produces the reconstructed attractor, $A^{\prime}$. If the reconstruction is accurate, then $A^{\prime}$ is the topological conjugate of the original attractor, $A$. Consequently, all dynamic properties of $A$ are projected to $A^{\prime}$. The criterion of the Takens Theorem (Takens, 1981) for a precise phase space reconstruction of an experimental trajectory dictates that $m$ must be greater then $[2 m c+1]$, where $m c$ is the estimated dimension of the attractor.

According to the Takens Theorem, this is efficient when the number of points of the timeseries, $N$, is infinite, meaning that for an infinite number of points, $A$ and $A^{\prime}$ have the same properties. However, for most experimental methods, $N$ is a finite number and in many cases is confined to 3000 4000 points. Therefore, only $A^{\prime}$ is estimated in the recon- structed space and retains only some of the properties of $A$ (not all). Essential to phase space reconstruction, especially for the Delay Times method, is the estimation of the time lag, $\tau$. There is a range of methods for estimating $\tau$, the most popular being the calculation of the decorrelation time.

The decorrelation time is calculated either from the first zero-value of the Autocorrelation function or from the first minimum value of the mutual information function. The $\mathrm{Au}-$ tocorrelation function has been described in the previous section. The mutual information method is widely accepted and it computes the nonlinear and linear correlation of the Autocorrelation function. Once the Autocorrelation function has been normalized, the decorrelation time is found from the smallest time lag for which the function tends to zero. Similarly, the decorrelation time can also be found from the smallest time lag for which the mutual information function tends to zero.

According to Broomhead and King (1986); Albano et al. (1988); Kugiumtzis (1996) the results of a time-series analysis depends on the window length $(m-1) \tau$, which incorporates both the embedding dimension $m$ and the time lag $\tau$. Therefore, the constraint to the above methods is the limit on the size of the window, $(m-1) \tau$. A proper value for the window size provides good phase space reconstruction and ensures that all the points of the reconstructed phase space come from the same trajectory. As mentioned above, the Takens theorem dictates that proper phase space reconstruction is achieved when $m$ is greater than [ $2 m c+1]$. This criterion is difficult to satisfy for increased values of $\tau$ due to the subsequently larger values of $(m-1) \tau$. A consistent window arises from the decorrelation time, seen as the time needed for the first decay of the Autocorrelation function. A time lag, $\tau$, is chosen and the reconstructed dynamics are embedded in the $m$-dimensional phase space.

After the phase space reconstruction of the system's assumed dynamics, non-linear dynamics algorithms are developed for the experimental analysis of a timeseries. The most popular algorithmic method is the Delay Times method, also known as the Algorithm of Grassberger and Procaccia (1983), which estimates the Correlation Dimension from the computation of the correlation integral.

The Grassberger Procaccia Algorithm (Grassberger and Procaccia, 1983) assumes a time-series, $X(i)$, which is a measure over time $i$ of a parameter of an $m$-dimensional dynamic system, for $i \in[1, N]$. The phase space reconstruction of this system is done according to the Takens theorem. Once again, the vector coordinates are constructed as in Eq. (8) and it is assumed that this vector is the trajectory vector of the $i$-th time point of the reconstructed phase space of the dynamic system. The whole trajectory is $\boldsymbol{x}(1), \boldsymbol{x}(2), \ldots, \boldsymbol{x}(i), P \ldots, \boldsymbol{x}(\rho)$ where $\rho=N-(m-1) \tau$. As mentioned and according to the Takens theorem, $A^{\prime}$ is the attractor to the reconstructed system dynamics and the topological conjugate to the original attractor $A$. Properties such as the Correlation Dimension are maintained after the projection of $A$ to $A^{\prime}$. The Correlation Dimension is defined 
as

$D_{2}=\lim _{N \rightarrow \infty} \frac{\log (C(m, r, \tau))}{\log (r)}$

where $r$ is a distance radius in the reconstructed phase space. The index 2 in $D_{2}$ is used because the Correlation Dimension is a special case of the generalized dimension $D_{q}$ where $q$ integer. $C(m, r, \tau)$ is the correlation integral and is defined as

$C(m, r, \tau)=\frac{2}{N-1} \sum_{i=1}^{N} \sum_{j=i+1}^{N} \Theta\left[r-\left\|\boldsymbol{x}_{i}-\boldsymbol{x}_{j}\right\|\right]$,

where $\boldsymbol{x}_{i}$ and $\boldsymbol{x}_{j}$ are as in Eq. (8). $\Theta$ is the Heavyside function:

$\Theta(i)=\left\{\begin{array}{l}1, \text { if } i \geq 0 \\ 0 \text { if } i \leq 0\end{array}\right.$

The Euclidean norm used in the above equation states that the difference between $\boldsymbol{x}_{i}$ and $\boldsymbol{x}_{j}$ is the maximum difference among their coordinates:

$$
\begin{aligned}
& \left\|\boldsymbol{x}_{i}-\boldsymbol{x}_{j}\right\|=\left\{|X(i)-X(j)|^{2}+|X(i+\tau)-X(j+\tau)|^{2}+\right. \\
& \left.\ldots+|X(i+(m-1) \tau)-X(j+(m-1) \tau)|^{2}\right\}^{\frac{1}{2}} .
\end{aligned}
$$

The formula Eq. (10) simply says: for specific $m, r, \tau$ find all pairs of $\boldsymbol{x}_{i}$ and $\boldsymbol{x}_{j}$ in the reconstructed time-series $\boldsymbol{x}(t)$ for which the distance $\left\|\boldsymbol{x}_{i}-\boldsymbol{x}_{j}\right\|$ is smaller than $r$.

According to this algorithm the $C(m, r, \tau)$ is computed for increasing values of $m$ and for a steady range of $r$. For each $\log (C)$ versus $\log (r)$ plot a scaling region is been selected and the slope of the curve is calculated for this scaling region with a simple method (i.e. least squares). If the slope values estimated for each $m$ converge in a steady value, then this steady value corresponds to the correlation dimension of the timeseries.

\section{Appendix B Approximate Entropy}

Given $N$ data points, $X(1), X(2), X(3), \ldots, X(N)$, the $\operatorname{ApEn}(m, r, N)$ is estimated, where $r$ is a threshold and $m$ a window size. The vector sequences necessary for phase space reconstruction, $\boldsymbol{x}(i)$, are constructed with $\boldsymbol{x}(N-m+1)$, defined by $\boldsymbol{x}(i)=[X(i), \ldots, X(i+m-1)]$. These vectors represent $m$ consecutive $X$ values, using the $i$-th point as the starting point. The distance $\|\boldsymbol{x}(i), \boldsymbol{x}(j)\|$ is defined between the vectors $\boldsymbol{x}(i)$ and $\boldsymbol{x}(j)$ as the infinity norm

$$
\begin{aligned}
& || X(i)-X(j)||=\max \{|X(i)-X(j)|, \\
& |X(i+1)-X(j+1)|, \\
& \ldots,|X(i+m-1)-X(j+m-1)|\} .
\end{aligned}
$$

The probability that $|X(i+m-1)-X(j+m-1)| \leq r$ given that $|X(i)-X(j)| \leq r$ and $|X(i+1)-X(j+1)| \leq r \quad$ and $|X(i+2)-X(j+2)| \leq r$ and $\ldots$ is true is termed $C_{r}^{m}(i)$, where, once again, $r$ is the a threshold and $m$ the window size. For example, if $m=2, C_{r}^{2}(i)$ for $i=1, \ldots, N$ is the probability that $|X(i+1)-X(j+1)| \leq r$ given that $|X(i)-X(j)| \leq r$.

The sequence in Eq. (14) is used to construct the $C_{i}^{m}(r)$ for each $i \leq N-m+1$ as in

$C_{r}^{m}(i)=$

$\frac{[\text { no.of } j \leq N-m+1, \text { suchthat }|| \boldsymbol{x}(i)-\boldsymbol{x}(j) \| \leq r]}{N-m+1}$

$\Phi^{m}(r)$ is defined as

$\Phi^{m}(r)=\frac{1}{N-m+1} \sum_{i=1}^{N-m+1} \ln C_{i}^{m}(r)$,

where $\ln$ is the natural logarithm. Then Approximate Entropy is defined as

$\operatorname{ApEn}(m, r)=\lim _{N \rightarrow \infty}\left[\Phi^{m}(r)-\Phi^{m+1}(r)\right]$.

It is therefore found that $-A p E n=\Phi^{m+1}(r)-\Phi^{m}(r)$ and is equal to the average over $i$ of the natural $\log$ of the conditional probability that $|X(j+m)-X(i+m)| \leq r$, given that $|X(j+k)-X(i+k)| \leq r$, for $k=0,1,2, \ldots, m-1$.

Several trials of this algorithm were run on the HRV data and it was adjusted accordingly in order to obtain a better distinction between the two subject groups. The first step in computing the Approximate Entropy is finding the length vector for $m=2$, which is $[X(i), X(i+1)]$, denoted $\boldsymbol{x}(i)$. All vectors that are close to $\boldsymbol{x}(i), \boldsymbol{x}(j)=[X(j), X(j+1)]$, are identified. As has already been stated, the vector $\boldsymbol{x}(j)$ is close to $\boldsymbol{x}(i)$ if $\|\boldsymbol{x}(i), \boldsymbol{x}(j)\| \leq r$. This, by definition, means that both $|X(i)-X(j)| \leq r$ and $|X(i+1)-X(j+1)| \leq r$ apply. A count of all the vectors $\boldsymbol{x}(j)$ close to $\boldsymbol{x}(i)$ is found and called $B$. The next step is to compute the rest of the $x(j)$ vectors for which $|X(i+2)-X(j+2)| \leq r$, and call it $A$. The ratio of $A / B$ represents the conditional probability that $X(j+2)$ is close to $X(i+2)$, given that the vector $\boldsymbol{x}(j)$ is close to $\boldsymbol{x}(i)$.

The above process is repeated for each length 2 vector $\boldsymbol{x}(i)$, calculating the conditional probability. The $A p E n$ is found by calculating the average of the logarithm of these conditional probabilities and taking its negative (to make it positive), as seen in Eq. (18)

$$
\begin{aligned}
& -A p E n=\Phi_{r}^{m+1}-\Phi_{r}^{m} \\
& =\left[\frac{1}{N-m} \sum_{i=1}^{N-m} \ln \left(C_{r}^{m+1}(i)\right)\right]- \\
& {\left[\frac{1}{N-m} \sum_{i=1}^{N-m+1} \ln \left(C_{r}^{m}(i)\right)\right]} \\
& \simeq \frac{1}{N-m} \sum_{i=1}^{N-m}\left[\ln \left(C_{r}^{m+1}(i)\right)-\ln \left(C_{r}^{m}(i)\right)\right] \\
& =\frac{1}{N-m} \sum_{i=1}^{N-m} \frac{\ln \left(C_{r}^{m+1}(i)\right)}{\ln \left(C_{r}^{m}(i)\right)}
\end{aligned}
$$


The calculation of the conditional probabilities will result in values between 0 and 1 . If the timeseries is regular, the values $X(i), X(i+1), X(i+2)$ are expected to be close to each other, as are $X(j), X(j+1), X(j+2)$. Therefore, the differences $|X(i)-X(j)|,|X(i+1)-X(j+1)|$ and $|X(i+2)-X(j+2)|$ will be close to each other for many values of $i, j$. This means that the conditional probabilities are expected to be closer to 1 for time-series coming from more regular processes. The negative logarithm of such a value will be closer to 0 .

Conversely, random processes will produce conditional probabilities closer to 0 , the negative logarithms of which will be closer to 1 . The comparison of subsequent vectors in a random signal will result in different values in the successive vector distances. Thus, the $A p E n$ values for signals coming from regular processes will be lower than the $A p E n$ values coming from random signals. In this application, this implies that low $A p E n$ values are to be clinically associated with cardiac pathology, while high values indicate a healthy and robust heart.

The previous algorithm calculates an estimation of the value of the Approximate Entropy, which is equal to the theoretical one, when the $N$ tends to infinity. An examination of this algorithm reveals that it is analogous to the Grassberger \& Procaccia Algorithm (the Delay Times method) for the Correlation Dimension estimation. Theoretical calculations by Wolf et al. (1965) indicate that reasonable estimations are achieved with an $N$ value of at least $10 \mathrm{~m}$ and preferably $30 \mathrm{~m}$. In the experimental analysis, $N=2000$ and $m=2$ were used, producing satisfactory statistical $A p E n$ validity.

However, the ApEn is a biased statistic. The expected value of $A p E n(m, r, N)$ increases asymptotically with $N$ to $A p \operatorname{En}(m, r)$ for all processes. The choice of window for each vector $\boldsymbol{x}$ is also important for the $A p E n$ estimation. However, the interest in this method is not in the reconstructed space, but rather in having a sufficient number of vectors in close proximity to each other, so that accurate conditional probabilities can be found. This work was partly supported by the PYTHAGORAS fellowships.

Edited by: M. Contadakis

Reviewed by: two referees

\section{References}

Albano, A., Muench, J., Schwartz, C., Mees, A., and Rapp, P.: Singular values decomposition and the Grassberger-Procaccia algorithm, Phys. Rev. A, 38, 3017-3026, 1988.

Alexeev, D. and Egorov, P.: Persistent cracks accumulation under loading of rocks and ccncentration criterion of failure, Reports of RAS 333, 6, 769-770, (in Russian), 1993.

Alexeev, D., Egorov, P., and Ivanov, V.: Hurst statistics of time dependence of electromagnetic emission under rocks loading, Physical-Technical problems of exploitation of treasures of the soil, 5, 27-30, (in Russian), 1993.

Bak, P.: How nature works, Oxford: Oxford UP, 1997.

Bak, P. and Tang, C.: Earthquakes as a self-organized critical phenomenon, J. Geophys. Res., 94, 15 635-15 637, 1989.
Bak, P., Tang, C., and Weisenfeld, K.: Self-organized criticality: an explanation of 1/f noise, Phys. Rev. A, 38, 364-374, 1987.

Beggs, J. and Plenz, D.: Neuronal avalanches in neocortical circuits, J. Neurosci, 23, 11 167-11 177, 2003.

Broomhead, D. and King, G.: Extracting qualitative dynamics from experimental data, Physica D, 20, 217-236, 1986.

Contoyiannis, Y., Diakonos, F., Kapiris, P., and Eftaxias, K.: Intermittent dynamics of critical pre-seismic electromagnetic fluctuations, Phys. Chem. Earth, 29, 397-408, 2004.

Dobrovolsky, I., Gershenzon, N., and Gokhberg, M.: Theory or Electrokinetic Effects occurring at the Final State in the preparation of a Tectonic Earthquake, Physics of the Earth and Planetary Interiors, 57, 144-156, 1989.

Eftaxias, K., Kopanas, J., Bogris, N., Kapiris, P., Antonopoulos, G., and Varotsos, P.: Detection of electromagnetic earthquake precursory signals in Greece, Proc. Japan Acad., 76(B), 45-50, 2000.

Eftaxias, K., Kapiris, P., Polygiannakis, J., Bogris, N., Kopanas, J., Antonopoulos, G., Peratzakis, A., and Hadjicontis, V.: Signatures of pending earthquake from electromagnetic anomalies, Geophys. Res. Lett., 28, 3321-3324, 2001.

Eftaxias, K., Kapiris, P., Dologlou, E., Kopanas, J., Bogris, N., Antonopoulos, G., Peratzakis, A., and Hadjicontis, V.: EM anomalies before the Kozani earthquake: A study of their behavior through laboratory experiments, Geophys. Res. Lett., 29, 69/1-69/4, 2002.

Eftaxias, K., Kapiris, P., Polygiannakis, J., Peratzakis, A., Kopanas, J., and Antonopoulos, G.: Experience of short term earthquake precursors with VLF-VHF electromagnetic emissions, Natural Hazards and Earth System Sciences, 3, 217-228, 2003,

SRef-ID: 1684-9981/nhess/2003-3-217.

Eftaxias, K., Frangos, P., Kapiris, P., Polygiannakis, J., Kopanas, J., Peratzakis, A., Skountzos, P., and Jaggard, D.: Review and a Model of Pre-Seismic electromagnetic emissions in terms of fractal electrodynamics, Fractals, 12, 243-273, 2004.

Farmer, A. and Swinney, H.: Independent coordinates for strange attractors from mutual information, Phys. Rev. A, 33, 11341140, 1986.

Fukuda, K., Nunes, L., and Stanley, H.: Similarities between communication dynamics in the Internet and the automatic nervous system, Europhys. Lett., 62, 189-195, 2003.

Gershenzon, N. and Bambakidis, G.: Modelling of seismoelectromagnetic phenomena, Russ. J. Earth Sci., 3, 247-275, 2001.

Gilden, D., Thornton, T., and Mallon, M.: $1 / f$ noise in human cognition, Science, 267, 1837-1839, 1995.

Goldberger, A., Amaral, L., Hausdorff, J., Ivanov, P., and Peng, C.K.: Fractal dynamics in physiology: Alterations with disease and aging, PNAS, 2466-2472, 2002.

Grassberger, P. and Procaccia, I.: Characterization of strange attractors, Phys. Rev. Lett., 50, 346-349, 1983.

Hayakawa, M.: Atmospheric and Ionospheric Electromagnetic Phenomena Associated with Earthquakes, Terrapub, Tokyo, 1999.

Hayakawa, M. and Fujinawa, Y.: Electromagnetic Phenomena Related to Earthquake Prediction, Terrapub, Tokyo, 1994.

Hayakawa, M. and Molchanov, O.: Seismo Electromagnetics, Terrapub, Tokyo, 2002.

Hayakawa, M., Ito, T., and Smirnova, N.: Fractal analysis of ULF geomagnetic data associated with the Guam eartquake on August 8, 1993, Geophys, Res. Lett., 26, 2797-2800, 1999.

Hayakawa, M., Itoh, T., Hattori, K., and Yumoto, K.: ULF electromagnetic precursors for an earthquake at Biak, Indonesia on 
February 17, 1996, Geophys. Res. Lett., 27(10), 1531-1534, 2000.

Heneghan, C. and McDarby, G.: Establishing the relation between detrended fluctuation analysis and power spectral density analysis for stohastic processes, Phys. Rev. E, 62, 6103-6110, 2000.

Herz, A. and Hopfield, J.: Earthquake cycles and neural reverberations: collective oscillations in systems with pulse-coupled threshold elements, Phys. Rev. Lett., 75, 1222-1225, 1995.

Hopfield, J.: Neurons, dynamics and computation, Physics Today, 40, 40-46, 1994.

Hristopulos, D.: Permissibility of fractal exponents and models of band-limited two-point functions for fGn and $\mathrm{fBm}$ random fields, Stohastic Environmental Research and Risk Assessment, 17, 191-216, 2003.

Hurst, H.: Long term storage capacity of reservoirs, Trans. Am. Soc. Civ. Eng., 116, 770-808, 1951.

Ivanov, P., Amaral, L., Goldberger, A., Havlin, S., Rosenblum, M., Struzik, Z., and Stanley, H.: Multifractality in human heartbeat dynamics, Nature, 399, 461-465, 1999.

Kaiser, G.: A. Friendly Guide to Wavelets, Birkhauser, 1994.

Kapiris, P., Polygiannakis, J., Nomicos, K., and Eftaxias, K.: VHFelectromagnetic evidence of the underlying pre-seismic critical stage, Earth Planets Space, 54, 1237-1246, 2002.

Kapiris, P., Eftaxias, K., Nomikos, K., Polygiannakis, J., Dologlou, E., Balasis, G., Bogris, N., Peratzakis, A., and Hadjicontis, V.: Evolving towards a critical point: A possible electromagnetic way in which the critical regime is reached as the rupture approaches, Nonlin. Proc. Geophys., 10, 1-14, 2003.

Kapiris, P., Balasis, G., Kopanas, J., Antonopoulos, G., Peratzakis, A., and Eftaxias, K.: Scaling similarities of multiple fracturing of solid materials, Nonlin. Proc. Geophys., 11, 137-151, 2004a, SRef-ID: 1607-7946/npg/2004-11-137.

Kapiris, P., Eftaxias, K., and Chelidze, T.: The electromagnetic signature of prefracture criticality in heterogeneous media, Phys. Rev. Lett., 92, 065 702/1-4, 2004b.

Karamanos, K., Nikolopoulos, S., and Hizanidis, K.: Block Entropy analysis of long recorded Electrocardiograms as a good way for discrimination between Normal subjects and Coronary patients, International Journal of Computing and Anticipatory systems (IJCAS, Liege, Proceedings of CASYS 2003), 2004.

Kugiumtzis, D.: State space reconstruction parameters in the analysis of c haotic time series - the role of the time window length, Physica D, 95, 13-28, 1996.

Li, X., Polygiannakis, J., Kapiris, P., Balasis, G., Peratzakis, A., Yao, X., and Eftaxias, K.: Evolving towards a biological or geophysical catastrophic event: emergence of isomorphic precursory alterations in scaling parameters in terms of intermitent criticality, in 4th International Conference on Fractals and Dynamics Systems in Geoscience, Kloster Seeon, Germany, May 19-22, Book of Abstract, 55-57, 2004.

Malamud, B., Morein, G., and Turcotte, D.: Forest fires: an example of self-oganized critical behavior, Science, 281, 1840-1842, 1998.

Mandelbrot, B. and Ness, J.: Fractional Brownian motions, fractional noises and applications, SIAM Rev., 10, 422-437, 1968.

Mantegna, R. and Stanley, H.: Scaling behavior in the dynamics of an economic index, Nature, 376, 46-49, 1995.

Maslov, S., Paczuski, M., and Bak, P.: Avalanches and 1/ $f$ Noise in Evolution and Growth Models, Phys. Rev. Lett., 73, 2162, 1994.

Meneveau, C. and Sreenivasan, K.: Simple multifractal cascade model for fully developed turbulance, Phys. Rev. Lett., 59, 14241427, 1987.
Mizutani, H. and Ishido, T.: A new interpretation of magnetic field variation associated with the Matsushiro earthquake occurrence, J. Geomagn. Geoelectr., 28, 179-188, 1976.

Nikolopoulos, S., Alexandridi, A., Nikolakeas, S., and Manis, G.: Experimental Analysis of Heart Rate Variability of LongRecording Electrocardiograms in Normal Subjects and Patients with Coronary Artery Disease and Normal Left Ventricular Function, J. Biomed. Inf., 36, 202-217, 2003.

Peng, C., Havlin, S., Stanley, H., and Goldberger, A.: Quantification of scaling exponents and crossover phenomena in nonstationary heartbeat timeseries, Chaos, 5, 82-87, 1995.

Pincus, S.: Approximate Entropy: A Complexity Measure for Biologic Time series Data, in The proceedings of IEEE 17th Annual Northeast Bioengineering Conference, pp. 35-36, New York: IEE Press, 1991.

Ponomarev, A., Zavyalov, A., Smirnov, V., and Lockner, D.: Physical modelling of the formation and evolution of seismically active fault zones, Tectonophysics, 277, 57-81, 1997.

Ramirez-Rojas, A., Pavia-Miller, C., and Angulo-Brown, F.: Statistical behavior of the spectral exponent and the correlation time of electric self-potential time series associated to the $\mathrm{Ms}=7.4$ September 14, 1995 earthquake in Mexico, Phys. Chem. Earth, 29, 305-312, 2004.

Rundle, J., Klein, W., Gross, S., and Turcotte, D.: Boltzmann fluctuations in numerical simulations of nonequilibrium lattice threshold systems, Phys. Rev. Lett., 75, 1658-1661, 1995.

Rundle, J., Tiampo, K., Klein, W., and Martins, J. S.: Selforganization in leaky threshold systems: the influence of nearmean field dynamics and its implications for earthquakes, neurology, and forecasting, PNAS, 99, 2514-2521, 2002.

Sethna, J., Dahmen, K., and Myers, C.: Crackling noise, Nature, 410, 242-250, 2001.

Sornette, D.: Predictability of catastrophic events: Material rupture, earthquakes, turbulence, financial crashes, and human birth, PNAS, 99, 2522-2529, 2002.

Stanley, H.: Scaling, universality, and renormalization: Three pillars of modern critical phenomena, Rev. Mod. Phys., 71, S358S366, 1999.

Stanley, H.: Exotic statistical physics: Applications to biology, medicine, and economics, Physica A, 285, 1-17, 2000.

Stanley, H., Amaral, L., Goldberger, A., Havlin, S., Ivanov, P., and Peng, C.: Statistical physics and physiology: Monofractal and multifractal approaches, Physica A, 106, 309-324, 1999.

Surkov, V., Uyeda, S., Tanaka, H., and Hayakawa, M.: Fractal properties of medium and seismoelectric phenomena, J. Geodynamics, 33, 477-487, 2002.

Takens, F.: Detecting strange attractors in turbulence, in Dynamical systems and turbulence, Warwick 1980: Proceedings of symposium held at the university of Warwick 1979-1980, SpringerVerlag, Berlin, 366-381, 1981.

Telesca, L. and Lapenna, V.: A new approach to investigate the correlation between geoelectrical time fluctuations and earthquakes in a seismic area of southern Italy, Geophys. Res. Lett., 28, 4375-4378, 2001.

Turcotte, D.: Fractals and chaos in geology and geophysics, Cambridge University Press, 1992.

Turcotte, D. and Rudle, J.: Self-organized complexity in the physical and social sciences, PNAS, 99, 2463-2465, 2002.

Usher, M., Stemmler, M., and Olami, Z.: Dynamic pattern formation leads to $1 / f$ noise in neural populations, Phys. Rev. Lett., 74, 326-329, 1995. 
Varotsos, P. and Alexopoulos, K.: Physical properties of the variation of the electric field of the earth preceding earthquakes, I, Tectonophysics, 110, 73-98, 1984a.

Varotsos, P. and Alexopoulos, K.: Physical properties of the variation of the electric field of the earth preceding earthquakes, II, Tectonophysics, 110, 99-125, 1984b.

Varotsos, P. and Sarlis, N.: A review of the recent VAN efforts: The explanation of the SES physical properties, in LithosphereAtmosphere-Ionosphere Coupling, Terrapub, Tokyo, 131-140, 2002.

Varotsos, P., Lazaridou, M., Eftaxias, M., ANtonopoulos, K., Makris, G., and Kopanas, J.: Short term earthquake prediction in Greece by seismic electric signals, in: A Critical Review of VAN, edited by: Lighthill, J., World Scientific, Singapore, 2976, 1996.
Vicsek, T.: Fractal Growth Phenomena, World Scientific, Singapore, 1993.

Vicsek, T.: A question of scale, Nature, 411, 421, 2001.

Vicsek, T.: The bigger picture, Nature, 418, 131, 2002.

Wolf, A., Swift, J., Swinney, H., and Vastano, J.: Determining Lyapunov exponents from a time series, Physica D, 16, 285-317, 1965.

Worrell, G., Cranstoun, S., Litt, B., and Echauz, J.: Evidence for self-organized criticality in human epileptic hippocampus, Neurophysiology, Basic and Clinical, 13, 1-6, 2002.

Zhao, X. and Chen, T.: Type of self-organized criticality model based on neural networks, Phys. Rev. E, 65, 026 114-1-026 114$6,2002$. 